\title{
Dirac and Faddeev-Jackiw quantization of a five-dimensional Stüeckelberg theory with a compact dimension
}

\author{
Alberto Escalante* and Moises Zarate† \\ Instituto de Física Luis Rivera Terrazas, Benemérita Universidad Autónoma de Puebla, \\ (IFUAP). Apartado postal J-48 72570 Puebla. Pue., México,
}

(Dated: June 27, 2018)

\begin{abstract}
A detailed Hamiltonian analysis for a five-dimensional Stüeckelberg theory with a compact dimension is performed. First, we develop a pure Dirac's analysis of the theory, we show that after performing the compactification, the theory is reduced to four-dimensional Stüeckelberg theory plus a tower of Kaluza-Klein modes. We develop a complete analysis of the constraints, we fix the gauge and we show that there are present pseudo-Goldstone bosons. Then we quantize the theory by constructing the Dirac brackets. As complementary work, we perform the Faddeev-Jackiw quantization for the theory under study, and we calculate the generalized Faddeev-Jackiw brackets, we show that both the Faddeev-Jackiw and Dirac's brackets are the same. Finally we discuss some remarks and prospects.
\end{abstract}

PACS numbers: 98.80.-k,98.80.Cq

\section{INTRODUCTION}

It is well know that four dimensional Proca's theory is not a gauge theory; the theory describes a massive vector field and the physical degrees of freedom are three, this is, the addition of a mass term to Maxwell theory breaks the gauge invariance of the theory and adds one physical degree of freedom to electromagnetic degrees of freedom [1 3 ]. However, in spite of Proca is not a gauge theory and it was believed by several people that only massless vector theories are gauge invariant, Stüeckelberg introduced to Proca's theory a scalar field converting the theory to be massive but preserving gauge invariance [4, [5]. The Stüeckelberg's mechanism consists in the introduction of new fields to reveal a symmetry of a gauge fixed theory. Moreover, Pauli showed that Stüeckelberg's formulation of a massive vector field satisfies a restricted $U(1)$ gauge invariance, similar to that one encountered in quantum electrodynamics [6, 7]. The studio of Stüeckelberg's Lagrangian becomes to be relevant in several contexts of theoretical physics, for instance, gauge bosons masses through Stüeckelberg couplings are present in string theory and supergravity [8], the mechanism turned out to be crucial in the covariant quantization of the spacetime supersymmetric string theory [9], and also Stüeckelberg fields were introduced as an essential tool for the formulation of the antisymmetric

*Electronic address: aescalan@ifuap.buap.mx

${ }^{\dagger}$ Electronic address: mzarate@ifuap.buap.mx 
partner to the graviton [10]. Furthermore, Stüeckelberg's mechanism provides an alternative way to the Higgs mechanism. In fact, Stüeckelberg's mechanism archive gauge symmetry braking without affecting renormalizability [11].

By taking into account the ideas explained above, in this paper we perform the canonical analysis and the Faddeev-Jackiw [FJ] quantization for a five-dimensional Stüeckelberg theory with a compact dimension. Nowadays, the study of models involving extra dimensions have an important activity in order to explain and solve some fundamental problems found in theoretical physics, such as, the problem of mass hierarchy, the explanation of dark energy, dark matter and inflation [12]. Furthermore, extra dimensions become also important in theories of unification trying of incorporating gravity and gauge interactions consistently, for instance, string theory and grand unification theories. Moreover, there are phenomenological and theoretical motivations to quantize a gauge theory in extra dimensions, for instance, if there exist extra dimensions, then their effects could be tested in the actual LHC collider, and in the International Linear Collider [13]. In this manner, the study of five-dimensional Stüeckelberg theory becomes relevant in the context of extra dimensions, in particular, we study the effects of the extra dimension on the theory when it is compactified on a $S^{1} / \mathbf{Z}_{2}$ orbifold. In this respect, let us show the relevance that the higher Kaluza-Klein [KK] modes of a vector field gain their masses through a Stüeckelberg's mechanism by performing the compactification on a circle. In fact, in [5] is reported the following action

$$
L_{5} D=-\frac{1}{4} \mathcal{F}_{I J}\left(x_{I}\right) \mathcal{F}^{I J}\left(x_{I}\right)-\frac{1}{2 \xi}\left(\partial_{I} \mathcal{A}^{I}\left(x_{I}\right)\right)^{2},
$$

where $x_{I}\left(x_{\mu}, y\right), x_{\mu}$ label the four-dimensional manifold and $y$ the fifth-spatial extra dimension, $\xi$ is as usual a gauge parameter, $\mathcal{A}_{I}=\left(A_{\mu}\left(x_{I}\right), \theta\left(x_{I}\right)\right)$ is the five-dimensional Abelian gauge field and $\theta\left(x_{I}\right)$ is the Stüeckelberg scalar. Now, we perform the expansion of the gauge field in harmonics, namely

$$
\begin{aligned}
A_{\mu}\left(x_{I}\right) & =\sum_{n=0}^{\infty} A_{\mu}^{(n)}\left(x_{\mu}\right) \zeta_{n}(y), \\
\theta\left(x_{I}\right) & =\sum_{n=0}^{\infty} \theta_{\mu}^{(n)}\left(x_{\mu}\right) \eta_{n}(y),
\end{aligned}
$$

where $\zeta$ and $\eta$ are harmonic functions on the interval $(0,2 \pi R)$. Thus, by considering that expansion and integrating over the fifth dimension we obtain the following four-dimensional Lagrangian

$L_{4 D}=\sum_{n=0}^{\infty}\left[-\frac{1}{4} \mathcal{F}_{\mu \nu}^{(n)} \mathcal{F}_{(n)}^{\mu \nu}-\frac{n^{2}}{2}\left(\frac{1}{R} A_{\mu}^{(n)}+n \partial_{\mu} \theta^{(n)}\right)^{2}-\frac{1}{2 \xi}\left(\left(\partial_{\mu} A^{\mu(n)}\right)^{2}+\frac{2 n}{R} \partial_{\mu} A^{\mu(n)} \theta^{(n)}+\left(\frac{1}{R}\right)^{2}\left(\theta^{(n)}\right)^{2}\right)\right]$

by choosing $\xi=1$ we find that the $\theta^{(n)}$ field decouple from the vector fields. Thus, we obtain one massless vector field and an infinite tower of massive vector fields, obtaining their mass by means the Stüeckelberg mechanism, and there is no Higgs mechanism involved in the generation of mass. Hence, our study will be focussed in the Stüeckelberg theory [4], we will perform the compactification on a $S^{1} / \mathbf{Z}_{2}$ orbifold and the theory under study will involve massive zero-modes, a tower of massive vector fields and pseudo-Goldstone bosons. Our analysis will be carry out by performing a pure 
Dirac's method [14 17]; we develop a full constraints program and we construct the Dirac brackets of the theory. On the other hand, we also perform the [FJ] symplectic formulation [18]; we show that Dirac's and generalized [FJ] brackets are the same. We apply the [FJ] formulation because it is a fundamental method for quantization; in fact, the [FJ] framework has been applied in several singular theories but it has not been applied for studying theories with compact dimensions, and we show in this paper that it is an elegant framework for annalyzying theories in this context.

The paper is organized as follows: In Sect. II, we study a five-dimensional Stüeckelberg theory, after performing the compactification process on a $S^{1} / \mathbf{Z}_{\mathbf{2}}$ orbifold we obtain a four-dimensional effective Lagrangian. We perform the Hamiltonian analysis and we obtain the complete constraints of the theory. By using the constraints, we show that under an appropriate fixed gauge, the fields $A_{5}^{(n)}$ are identified as pseudo-Goldston bosons, just like it appears in five-dimensional Maxwell theory. In Sect.III, we compute the Dirac brackets of the zero-modes. In Sect. IV, we calculate the Dirac brackets of the Kaluza-Klein $[\mathrm{KK}]$ modes . In Sect. V, we perform the [FJ] method; we construct the generalized $[\mathrm{FJ}]$ brackets and we show that the obtained [FJ] and Dirac's brackets coincide for the zero-modes. In Sect. VI, we calculate the generalized [FJ] brackets for the KK-modes; we show the equivalence among $[\mathrm{FJ}]$ and Dirac's brackets. In Sect. VII, we present some remarks and prospects.

\section{DIRAC'S ANALYSIS FOR FIVE-DIMENSIONAL STÜECKELBERG THEORY WITH A COMPACT DIMENSION}

The action that we shall study in this section is given by the following five-dimensional Stüeckelberg theory [4]

$$
S\left[A\left(x_{\mu}, y\right), \theta\left(x_{\mu}, y\right)\right]=\int d^{4} x \int_{0}^{2 \pi R} d y\left\{-\frac{1}{4} F^{M N} F_{M N}+m^{2}\left(A_{M}+\partial_{M} \theta\right)\left(A^{M}+\partial^{M} \theta\right)\right\},
$$

here $\theta$ is the Stüeckelberg scalar. It is important to remark, that the action is invariant under the gauge transformations

$$
\begin{aligned}
A_{M}(x, y) & \rightarrow A_{M}(x, y)-\partial_{M} \epsilon(x, y), \\
\theta(x, y) & \rightarrow \theta(x, y)+\epsilon(x, y),
\end{aligned}
$$

and the compactification will be performed in order to do not damage that gauge symmetry.

The notation that we will use along the paper is the following; the capital latin indices $M, N$ run over 0,1,2,3,5 here 5 label the extra compact dimension and these indices can be raised and lowered by the five-dimensional Minkowski metric $\eta_{M N}=(1,-1,-1,-1,-1) ; y$ will represent the coordinate in the compact dimension and $\mu, \nu=0,1,2,3$ are spacetime indices, $x^{\mu}$ the coordinates that label the points for the four-dimensional manifold $M_{4}$; furthermore we will suppose that the compact dimension is a $S^{1} / \mathbf{Z}_{\mathbf{2}}$ orbifold whose radius is $R$. In this respect, it is well knew that a simple 
compact one dimensional manifold is a circle $S^{1}$, just as was developed the compactification above. However, if we demand an additional reflection symmetry $Z_{2}$ with respect to the origin $y=0$, then we obtain an orbifold $S^{1} / Z_{2}$ which turns out to be important in the study of higher dimensional physics. The point $y=0$ is a fixed point because it is $Z_{2}$ invariant, and also $-\pi R$ is a second fixed point of the orbifold, thus, we can observe that $S^{1} / Z_{2}$ reduces to $S^{1}$ to line segment with fixed endpoints at $y=0$ and $y=\pi R$. Hence, the periodic boundary conditions on $S^{1}$ indicate that any dynamical field on $S^{1} / Z_{2}$ must be expanded in terms of functions with period 0 to $2 \pi R$. Therefore, the compactification on $S_{1} / Z_{2}$ reflects certain restrictions on the fields and by taking account the gauge symmetry (4), we requires that the fields satisfy the following [19]

$$
\begin{aligned}
A_{M}(x, y) & =A_{M}(x, y+2 \pi R), \\
A_{\mu}(x, y) & =A_{\mu}(x,-y), \\
A_{5}(x, y) & =-A_{5}(x,-y), \\
\theta(x, y) & =\theta(x, y+2 \pi R), \\
\theta(x, y) & =\theta(x,-y) .
\end{aligned}
$$

It is important to comment that for $S^{1}$ we expanded the fields in terms of complex exponentials, however, in that expansion there are mixtures of even and odd functions and this is not an appropriate basis for $S^{1} / Z_{2}$. Nevertheless, the dynamical variables defined on $M_{4} \times S^{1} / \mathbf{Z}_{2}$ can be expanded in terms of the complete set of harmonics [19 21]

$$
\begin{aligned}
& A_{5}(x, y)=\frac{1}{\sqrt{\pi R}} \sum_{n=1}^{\infty} A_{5}^{(n)}(x) \sin \left(\frac{n y}{R}\right) \\
& A_{\mu}(x, y)=\frac{1}{\sqrt{2 \pi R}} A_{\mu}^{(0)}(x)+\frac{1}{\sqrt{\pi R}} \sum_{n=1}^{\infty} A_{\mu}^{(n)}(x) \cos \left(\frac{n y}{R}\right) \\
& \theta(x, y)==\frac{1}{\sqrt{2 \pi R}} \theta^{(0)}(x)+\frac{1}{\sqrt{\pi R}} \sum_{n=1}^{\infty} \theta^{(n)}(x) \cos \left(\frac{n y}{R}\right) .
\end{aligned}
$$

For this theory, the dynamical variables for the zero mode are given by $A_{i}^{(0)}, A_{0}^{(0)}, \theta^{(0)}$ and for the KK-modes are $A_{5}^{(n)}, A_{i}^{(n)}, A_{0}^{(n)}, \theta^{(n)}$ with $i, j=1,2,3$. We shall suppose that the number of KKmodes is $k$, and we will take the limit $k \rightarrow \infty$ at the end of the calculations, thus, $n=1,2,3 \ldots k-1$. Let us develop the Hamiltonian analysis for the action (3); hence, we perform the $4+1$ decomposition and the compactification process, thus, the Lagrangian density takes the following form

$$
\begin{aligned}
\mathcal{L}= & -\frac{1}{4} F_{\mu \nu}^{(0)} F_{(0)}^{\mu \nu}+m^{2}\left(A_{\mu}^{(0)}+\partial_{\mu} \theta^{(0)}\right)\left(A_{(0)}^{\mu}+\partial^{\mu} \theta_{(0)}\right)+\sum_{n=1}^{\infty}\left[-\frac{1}{4} F_{\mu \nu}^{(n)} F_{(n)}^{\mu \nu}\right. \\
& +m^{2}\left(A_{\mu}^{(n)}+\partial_{\mu} \theta^{(n)}\right)\left(A_{(n)}^{\mu}+\partial^{\mu} \theta_{(n)}\right)+\frac{1}{2}\left(\partial_{\mu} A_{5}^{(n)}+\frac{n}{R} A_{\mu}^{(n)}\right)\left(\partial^{\mu} A_{(n)}^{5}+\frac{n}{R} A_{(n)}^{\mu}\right) \\
& \left.-m^{2}\left(A_{5}^{(n)}-\frac{n}{R} \theta^{(n)}\right)\left(A_{5}^{(n)}-\frac{n}{R} \theta_{(n)}\right)\right]
\end{aligned}
$$

where $F_{\mu \nu}^{(0)}$ and $F_{\mu \nu}^{(n)}$ are the field strength associated with the fields $A_{\mu}^{(0)}$ and $A_{\mu}^{(m)}$ respectively. To proceed with the canonical analysis, we define the momenta $\left(\Pi_{(n)}^{M}, P_{(n)}\right)$ conjugate to the fields 
$\left(A_{M}^{(n)}, \theta^{(n)}\right)$ respectively in the usual way

$$
\Pi_{(n)}^{M}=\frac{\delta L}{\delta \dot{A}_{M}^{(n)}}, \quad P_{(n)}=\frac{\delta L}{\delta \dot{\theta}^{(n)}},
$$

hence

$$
\begin{aligned}
& \Pi_{(0)}^{0}=0, \\
& \Pi_{(0)}^{i}=\partial_{0} A_{i}^{(0)}-\partial_{i} A_{0}^{(0)}, \\
& P_{(0)}=2 m^{2}\left(A_{0}^{(0)}+\partial_{0} \theta^{(0)}\right), \\
& \Pi_{(n)}^{0}=0, \\
& \Pi_{(n)}^{i}=\partial_{0} A_{i}^{(n)}-\partial_{i} A_{0}^{(n)}, \\
& \Pi_{(n)}^{5}=\partial_{0} A_{5}^{(n)}+\frac{n}{R} A_{0}^{(n)}, \\
& P_{(n)}=2 m^{2}\left(A_{0}^{(n)}+\partial_{0} \theta^{(n)}\right) .
\end{aligned}
$$

It is straightforward observe that the Hessian of the the action (6) is singular, the rank of Hessian is $5 k-6$ and there is $k$ null vectors. Thus, from the null vectors, we obtain the following $k$ primary constraints

$$
\begin{aligned}
& \phi_{(0)}^{0}: \Pi_{(0)}^{0} \approx 0, \\
& \phi_{(n)}^{0}: \Pi_{(n)}^{0} \approx 0 .
\end{aligned}
$$

The canonical Hamiltonian is obtained by the Legendre transformation as

$$
\begin{aligned}
H_{c}= & \int d^{3} x\left(\frac{1}{2} \Pi_{(0)}^{i} \Pi_{(0)}^{i}+\frac{1}{4 m^{2}} P_{(0)} P_{(0)}+\frac{1}{4} F_{i j}^{(0)} F_{(0)}^{i j}-A_{0}^{(0)}\left(\partial_{i} \Pi_{(0)}^{i}+P_{(0)}\right)-m^{2}\left(A_{i}^{(0)}+\partial_{i} \theta^{(0)}\right)^{2}\right. \\
& +\sum_{n=1}^{\infty}\left[\frac{1}{2} \Pi_{(n)}^{i} \Pi_{(n)}^{i}+\frac{1}{4 m^{2}} P_{(n)} P_{(n)}+\frac{1}{4} F_{i j}^{(n)} F_{(n)}^{i j}-A_{0}^{(n)}\left(\partial_{i} \Pi_{(n)}^{i}+\frac{n}{R} \Pi_{(n)}^{5}+P_{(n)}\right)-m^{2}\left(A_{i}^{(n)}+\partial_{i} \theta^{(n)}\right)^{2}\right. \\
& \left.\left.+\frac{1}{2} \Pi_{5}^{(n)} \Pi_{5}^{(n)}+\frac{1}{2}\left(\partial_{i} A_{5}^{(n)}+\frac{n}{R} A_{i}^{(n)}\right)^{2}+m^{2}\left(A_{5}^{(n)}-\frac{n}{R} \theta^{(n)}\right)^{2}\right]\right)
\end{aligned}
$$

the addition of primary constraints to the canonical Hamiltonian allows us to identify the primary Hamiltonian

$$
H_{P}=H_{c}+\int d x^{3}\left[\lambda_{(0)}^{(0)} \phi_{(0)}^{0}+\sum_{n=1}^{\infty} \lambda_{0}^{(n)} \phi_{(n)}^{0}\right]
$$

The non-vanishing fundamental Poisson brackets are given by

$$
\begin{aligned}
\left\{A_{M}^{(n)}\left(x^{0}, x\right), \Pi_{(n)}^{N}\left(x^{0}, z\right)\right\} & =\delta^{N}{ }_{M} \delta^{3}(x-z), \\
\left\{\theta^{(n)}\left(x^{0}, x\right), P_{(n)}\left(x^{0}, z\right)\right\} & =\delta^{3}(x-z) .
\end{aligned}
$$

Now, we need identify if the theory presents secondary constraints; for this aim, we calculate consistency among the primary constraints, thus, we obtain the following secondary constraints

$$
\begin{aligned}
& \dot{\phi}_{(0)}^{0}=\left\{\phi_{(0)}^{0}(x), H_{P}\right\} \approx 0 \quad \Rightarrow \quad \psi_{(0)}=\partial_{i} \Pi_{(0)}^{i}+P_{(0)} \approx 0, \\
& \dot{\phi}_{(n)}^{0}=\left\{\phi_{(n)}^{0}(x), H_{P}\right\} \approx 0 \quad \Rightarrow \quad \psi_{(n)}=\partial_{i} \Pi_{(n)}^{i}+\frac{n}{R} \Pi_{(n)}^{5}+P_{(n)} \approx 0 .
\end{aligned}
$$


On the other hand, from consistency of secondary constraints, does not emerge more constraints. In this way, with all the constraints at hand we need to identify which ones correspond to first and second class. For this aim, we compute the Poisson brackets between the primary and secondary constraints. We find that the Poisson brackets between primary and secondary constraints are computed as

$$
\begin{aligned}
\left\{\psi^{(0)}(y), \phi_{(0)}^{0}(x)\right\} & =0, \\
\left\{\psi^{(n)}(y), \phi_{(m)}^{0}(x)\right\} & =0,
\end{aligned}
$$

we observe that the Poisson brackets between primary with secondary constraints vanish, hence, the constraints of the theory under study are all first class constraints. In fact, there are 2 first class constraints for the zero-mode given by

$$
\begin{aligned}
& \gamma_{(0)}^{0}=\Pi_{(0)}^{0} \approx 0, \\
& \gamma^{(0)}=\partial_{i} \Pi_{(0)}^{i}+P_{(0)} \approx 0,
\end{aligned}
$$

where the second constraint of (15) is identified as the Gauss constraint for the zero-mode of conventional Stüeckelberg theory. Furthermore, there are $2 k-2$ first class constraints for the KK-modes given by

$$
\begin{aligned}
& \gamma_{(n)}^{0}=\Pi_{(n)}^{0} \approx 0, \\
& \gamma^{(n)}=\partial_{i} \Pi_{(n)}^{i}+\frac{n}{R} \Pi_{(n)}^{5}+P_{(n)} \approx 0,
\end{aligned}
$$

where the second constraint of (16) can be identified as the Gauss constraint for the excited modes. In this manner, we perform the counting of physical degrees of freedom as follows; there are $12 k-2$ dynamical variables and $2 k$ independent first class constraints, thus

$$
\begin{aligned}
\text { Number of physical degrees of freedom } & =\frac{1}{2}(12 k-2-2(2 k)) \\
& =4 k-1,
\end{aligned}
$$

we observe that if $k=1$, then we obtain 3 physical degrees of freedom corresponding to the physical degrees of freedom for the Stüeckelberg theory without a compact dimension, these degrees of freedom are associated with the zero-mode as is expected [4 6 ].

Moreover, by using the first class constraints obtained in (15), (16) we find the extended action

$$
\begin{aligned}
& S_{E}\left(Q_{K}, P_{K}, \lambda_{K}\right)=\int\left[\dot{A}_{\nu}^{(0)} \Pi_{(0)}^{\nu}+\dot{\theta}^{(0)} P_{(0)}-\mathcal{H}^{(0)}-\beta_{(0)} \gamma^{(0)}-\lambda_{0}^{(0)} \gamma_{(0)}^{0}+\sum_{n=1}^{\infty}\left\{\dot{A}_{N}^{(n)} \Pi_{(n)}^{N}+\dot{\theta}^{(n)} \Pi_{(n)}\right.\right. \\
& \left.\left.-\mathcal{H}^{(n)}-\lambda_{0}^{(n)} \gamma_{(n)}^{0}-\beta_{(n)} \gamma^{(n)}\right\}\right] d x^{3},
\end{aligned}
$$

where $Q_{K}$ y $P_{K}$ represent all the dynamical variables and their canonical momenta respectively, $\lambda_{K}$ stand for all Lagrange multipliers associated with the first class constraints. From the extended action, we identify the extended Hamiltonian given by

$$
H_{e x t}=H_{c}+\int\left[\beta_{(0)} \gamma^{(0)}+\lambda_{(0)}^{0} \gamma_{0}^{(0)}+\sum_{n=1}^{\infty}\left\{\lambda_{0}^{(n)} \gamma_{(n)}^{0}+\beta_{(n)} \gamma^{(n)}\right\}\right] d x^{3} .
$$


Now, we will calculate the gauge transformations on the phase space. For this important step, we use the first class constraints and we define the following gauge generator

$$
G=\int_{\Sigma}\left[\varepsilon_{(n)}^{0} \gamma_{0}^{(n)}+\varepsilon_{(n)} \gamma^{(n)}+\varepsilon_{(0)}^{0} \gamma_{0}^{(0)}+\varepsilon_{(0)} \gamma^{(0)}\right] d x^{3},
$$

thus, we find that the gauge transformations on the phase space for the zero-mode given by

$$
\begin{aligned}
\delta A_{\mu}^{(0)} & =-\partial_{\mu} \varepsilon^{(0)}, \\
\delta \theta^{(0)} & =\varepsilon^{(0)},
\end{aligned}
$$

and the gauge transformation for the KK-modes takes the form

$$
\begin{aligned}
\delta A_{\mu}^{(n)} & =-\partial_{\mu} \varepsilon^{(n)}, \\
\delta A_{5}^{(n)} & =\frac{n}{R} \varepsilon^{(n)}, \\
\delta \theta^{(n)} & =\varepsilon^{(n)},
\end{aligned}
$$

we can observe that this result is in agreement with the transformations (4). On the other hand, from the gauge transformations (21) we can consider the particular gauge fixing defined by $\varepsilon^{(n)}=-\frac{R}{n} A_{5}^{(n)}$. By using this gauge, the effective action given in (6) is reduced to

$$
\begin{aligned}
\mathcal{L}= & -\frac{1}{4} F_{\mu \nu}^{(0)} F_{(0)}^{\mu \nu}+m^{2}\left(A_{\mu}^{(0)}+\partial_{\mu} \theta^{(0)}\right)\left(A_{(0)}^{\mu}+\partial^{\mu} \theta_{(0)}\right)+\sum_{n=1}^{\infty}\left[-\frac{1}{4} F_{\mu \nu}^{(n)} F_{(n)}^{\mu \nu}+\left(m^{2}+\frac{n^{2}}{2 R^{2}}\right) A_{\mu}^{(n)} A_{(n)}^{\mu}\right. \\
& \left.+2 m^{2} A_{\mu}^{(n)} \partial^{\mu} \theta_{(n)}+m^{2} \partial_{\mu} \theta^{(n)} \partial^{\mu} \theta_{(n)}-\frac{m^{2} n^{2}}{R^{2}} \theta^{(n)} \theta_{(n)}\right]
\end{aligned}
$$

where we are able to observe that the KK-modes are massive fields, and $A_{5}^{(n)}$ has been absorbed and identified as pseudo-Goldstone bosons, just like is present in free five-dimensional Maxwell theory [19, 20]. Therefore, the five dimensional Stüeckelberg theory with a compact dimension, is composed by a four-dimensional Stüeckelberg theory associated with the zero-mode, a tower of KK-modes $A_{\mu}^{(n)}$ of mass $m^{2}+\frac{n^{2}}{2 R^{2}}$, and a tower of massive KK-modes $\theta^{(n)}$ of mass $\frac{m^{2} n^{2}}{R^{2}}$ plus interactive terms.

In the following sections, we will quantize the theory by constructing the Dirac brackets, then we will perform the $[\mathrm{FJ}]$ quantization of systems with constraints, and we shall prove that Dirac's and the generalized [FJ] brackets are the same. We will find that the advantage for applying the [FJ] is that there are less steps for arriving to the generalized brackets in comparison with Dirac's method, all this will be explained along the paper. 


\section{DIRAC'S BRACKET FOR THE ZERO-MODES}

In this section we will quantize the theory. By following with Dirac's formalism, the first class constraints obtained for the zero-mode and the KK-modes are given by

$$
\begin{aligned}
& \gamma_{(0)}^{0}=\Pi_{(0)}^{0} \approx 0, \\
& \gamma^{(0)}=\partial_{i} \Pi_{(0)}^{i}+P_{(0)} \approx 0, \\
& \gamma_{(n)}^{0}=\Pi_{(n)}^{0} \approx 0, \\
& \gamma_{(n)}=\partial_{i} \Pi_{(n)}^{i}+\frac{n}{R} \Pi_{(n)}^{5}+P_{(n)} \approx 0,
\end{aligned}
$$

since the zero-mode and the KK-modes are not coupled in both the Lagrangian and the constraints, then we can construct the Dirac brackets independently for each case, namely, first we will construct the Dirac brackets for the zero-mode, then we will construct the brackets for the KK-modes.

In order to construct the Dirac brackets for the zero-mode, we work with the following fixed gauge $\partial^{i} A_{i}^{(0)} \approx 0$ and $A_{0}^{(0)} \approx 0$, obtaining the following set of constraints

$$
\begin{aligned}
\chi_{1}^{(0)} & =\partial^{i} A_{i}^{(0)} \approx 0, \\
\chi_{2}^{(0)} & =\partial_{i} \Pi_{(0)}^{i}+P_{(0)} \approx 0, \\
\chi_{3(0)}^{0} & =\Pi_{(0)}^{0} \approx 0, \\
\chi_{4(0)}^{0} & =A_{0}^{(0)} \approx 0,
\end{aligned}
$$

under these gauge, now the constraints are all of second class. So, the $4 \times 4$ matrix whose entries are formed by the Poisson brackets among the constraints (24), namely $C_{\alpha \beta}$, is given by

$$
\begin{aligned}
C_{\alpha \beta}= & \left(\begin{array}{cccc}
\left\{\chi_{1}^{(0)}(x), \chi_{1}^{(0)}(y)\right\} & \left\{\chi_{1}^{(0)}(x), \chi_{2}^{(0)}(y)\right\} & \left\{\chi_{1}^{(0)}(x), \chi_{3}^{(0)}(y)\right\} & \left\{\chi_{1}^{(0)}(x), \chi_{4}^{(0)}(y)\right\} \\
\left\{\chi_{2}^{(0)}(x), \chi_{1}^{(0)}(y)\right\} & \left\{\chi_{2}^{(0)}(x), \chi_{2}^{(0)}(y)\right\} & \left\{\chi_{2}^{(0)}(x), \chi_{3}^{(0)}(y)\right\} & \left\{\chi_{2}^{(0)}(x), \chi_{4}^{(0)}(y)\right\} \\
\left\{\chi_{3}^{(0)}(x), \chi_{1}^{(0)}(y)\right\} & \left\{\chi_{3}^{(0)}(x), \chi_{2}^{(0)}(y)\right\} & \left\{\chi_{3}^{(0)}(x), \chi_{3}^{(0)}(y)\right\} & \left\{\chi_{3}^{(0)}(x), \chi_{4}^{(0)}(y)\right\} \\
\left\{\chi_{4}^{(0)}(x), \chi_{1}^{(0)}(y)\right\} & \left\{\chi_{4}^{(0)}(x), \chi_{2}^{(0)}(y)\right\} & \left\{\chi_{4}^{(0)}(x), \chi_{3}^{(0)}(y)\right\} & \left\{\chi_{4}^{(0)}(x), \chi_{4}^{(0)}(y)\right\}
\end{array}\right) \\
= & \begin{array}{c}
\chi_{1}^{(0)}(x) \\
\chi_{2}^{(0)}(x) \\
\chi_{3}^{(0)}(x)
\end{array}\left(\begin{array}{cccc}
0 & \chi_{2}^{(0)}(y) & \chi_{3}(y)^{(0)} & \chi_{4}^{(0)}(y) \\
& \chi_{4}^{(0)}(x)
\end{array} \quad \begin{array}{cccc}
0 & 0 & 0 & 0 \\
0 & 0 & 0 & -1
\end{array}\right) \delta^{3}(x-y),
\end{aligned}
$$

and its inverse takes the following form

$$
C^{\alpha \beta}=\left(\begin{array}{cccc}
0 & \frac{1}{-\nabla^{2}} & 0 & 0 \\
\frac{1}{\nabla^{2}} & 0 & 0 & 0 \\
0 & 0 & 1 & 0 \\
0 & 0 & 0 & -1
\end{array}\right) \delta^{3}(x-y)
$$


In this manner, the Dirac brackets of two functionals $F, G$ defined on the phase space, is expressed by

$$
\{F(x), G(z)\}_{D} \equiv\{F(x), G(z)\}-\int d^{2} u d^{2} w\left\{F(x), \xi_{\alpha}(u)\right\} C^{\alpha \beta}\left\{\xi_{\beta}(w), G(z)\right\},
$$

where $\{F(x), G(z)\}$ is the Poisson bracket between two functionals $F, G$, and $\xi_{\alpha}=\left(\xi_{1}, \xi_{2}, \xi_{3}, \xi_{4}\right)$ represent the set of second class constraints. By using this fact, we obtain the following Dirac's brackets for the zero-mode

$$
\begin{aligned}
\left\{A_{i}^{(0)}(x), \Pi_{(0)}^{j}(z)\right\}_{D} & =\left(\delta^{j}{ }_{i}-\frac{\partial^{j} \partial_{i}}{\nabla^{2}}\right) \delta^{3}(x-z), \\
\left\{P_{(0)}(x), \theta^{(0)}(z)\right\}_{D} & =-\delta^{3}(x-z), \\
\left\{\Pi_{(0)}^{i}(x), \theta^{(0)}(z)\right\}_{D} & =\frac{\partial^{i}}{\nabla^{2}} \delta^{3}(x-z),
\end{aligned}
$$

these are the Dirac brackets for a four-dimensional Stüeckelberg theory [22].

\section{DIRAC'S BRACKETS FOR THE KK-MODES}

Now, we will calculate the Dirac brackets for the KK-modes. For this aim, we observe that the gauge transformations given in (21), allow us to work with the following axial gauge $A_{5}^{(n)} \approx 0$ and $\Pi_{5}^{(n)}+\frac{n}{R} A_{0}^{(n)} \approx 0$. Thus, the set of constraints for the KK-modes are of second class given by

$$
\begin{aligned}
\chi_{1}^{(n)} & =A_{5}^{(n)} \approx 0, \\
\chi_{2(n)} & =\partial_{i} \Pi_{(n)}^{i}+\frac{n}{R} \Pi_{(n)}^{5}+P_{(n)} \approx 0, \\
\chi_{3(n)} & =\Pi_{(n)}^{0} \approx 0, \\
\chi_{4}^{(n)} & =\Pi_{5}^{(n)}+\frac{n}{R} A_{0}^{(n)} \approx 0 .
\end{aligned}
$$

Hence, the $4 \times 4$ matrix, namely $C_{\alpha \beta}^{(n)}$, whose entries are given by the Poisson brackets among the constraints (27) is given by

$$
\begin{aligned}
& \chi_{1}^{(n)}(y) \quad \chi_{2}^{(n)}(y) \quad \chi_{3}^{(n)}(y) \quad \chi_{4}^{(n)}(y) \\
& C_{\alpha \beta}^{(n)}=\begin{array}{c}
\chi_{1}^{(n)}(x) \\
\chi_{2}^{(n)}(x) \\
\chi_{3}^{(n)}(x) \\
\chi_{4}^{(n)}(x)
\end{array}\left(\begin{array}{cccc}
0 & \frac{n}{R} & 0 & 1 \\
-\frac{n}{R} & 0 & 0 & 0 \\
0 & 0 & 0 & -\frac{n}{R} \\
-1 & 0 & \frac{n}{R} & 0
\end{array}\right) \delta^{3}(x-z),
\end{aligned}
$$

and its inverse takes the following form

$$
\left(C_{\alpha \beta}^{(n)}\right)^{-1}=\left(\begin{array}{cccc}
0 & -\frac{R}{n} & 0 & 0 \\
\frac{R}{n} & 0 & 1 & 0 \\
0 & -1 & 0 & \frac{R}{n} \\
0 & 0 & -\frac{R}{n} & 0
\end{array}\right) \delta^{3}(x-z)
$$


Therefore, by using the matrix $\left(C_{\alpha \beta}^{(n)}\right)^{-1}$ and the definition of Dirac's brackets, the non-zero brackets among the physical fields of the KK-modes are given by

$$
\begin{aligned}
\left\{A_{i}^{(n)}(x), \Pi_{(n)}^{j}(z)\right\}_{D} & =\delta^{j}{ }_{i} \delta^{3}(x-z), \\
\left\{\Pi_{(n)}^{5}(x), A_{i}^{(n)}(z)\right\}_{D} & =\frac{R}{n} \partial_{i} \delta^{3}(x-z) \\
\left\{\theta^{(n)}(x), P_{(n)}(z)\right\}_{D} & =\delta^{3}(x-z),
\end{aligned}
$$

It is important to remark that these results are absent in the literature. On the other hand, in the following section, we will use the [FJ] quantization; we will observe that in [FJ] framework the gauge $A_{5}^{(n)} \approx 0$ will be interpreted as a constraint of the theory. Moreover, in such framework the gauge $\Pi_{5}^{(n)}+\frac{n}{R} A_{0}^{(n)}$ will not be invoked.

\section{FADDEEV-JACKIW QUANTIZATION FOR THE ZERO-MODE}

In this section we will perform the [FJ] framework for the action given in ([6]), and we will obtain by a different way the brackets given in (26) and (28), where they were obtained by using a pure Dirac's method. It is important to comment that the [FJ] formulation has not been applied for theories in the context of extra dimensions, thus, in this section we perform this formulation and we will show the advantages of the method.

For our purposes, first we will work with the zero-mode, thus, from the Legendre transformation (10) we identify the first order symplectic Lagrangian for the zero-mode given by

$$
\mathcal{L}^{0}=\Pi_{(0)}^{i} \dot{A}_{i}^{(0)}+P_{(0)} \dot{\theta}^{(0)}-V^{0}
$$

where $V^{0}=\left(\frac{1}{2} \Pi_{(0)}^{i} \Pi_{i}^{(0)}+\frac{1}{4 m^{2}} P_{(0)} P^{(0)}+\frac{1}{4} F_{i j}^{(0)} F_{(0)}^{i j}-A_{0}^{(0)}\left(\partial_{i} \Pi_{(0)}^{i}+P_{(0)}\right)-m^{2}\left(A_{i}^{(0)}+\partial_{i} \theta^{(0)}\right)^{2}\right)$. The corresponding symplectic equations of motion are given by [18]

$$
f_{i j}^{0} \dot{\xi}^{j}=\frac{\partial V^{0}}{\partial \xi^{i}}
$$

where the symplectic matrix is defined by

$$
f_{i j}^{0}(x, y)=\frac{\delta a_{j}(x)}{\delta \xi^{i}(y)}-\frac{\delta a_{i}(x)}{\delta \xi^{j}(y)} .
$$

Thus, from the symplectic Lagrangian (29), we identify the following set of symplectic variables as $\xi^{i}=\left(A_{i}^{(0)}, \Pi_{(0)}^{i}, \theta^{(0)}, P_{(0)}, A_{0}^{(0)}\right)$ and the components of the symplectic 1-forms are $a_{i}=$ $\left(\Pi_{(0)}^{i}, 0, P_{(0)}, 0,0\right)$. In this manner, by using the symplectic variables we obtain the following symplectic matrix

$$
f_{i j}^{0}(x, y)=\left(\begin{array}{ccccc}
0 & -\delta_{j}{ }^{i} & 0 & 0 & 0 \\
\delta_{j}{ }^{i} & 0 & 0 & 0 & 0 \\
0 & 0 & 0 & -1 & 0 \\
0 & 0 & 1 & 0 & 0 \\
0 & 0 & 0 & 0 & 0
\end{array}\right) \delta^{3}(x-y)
$$


where we can observe that this matrix is singular. In fact, in [FJ] framework, this means that there are constraints for the theory. We calculate the modes of this matrix; for this theory there is a mode and it is given by $v^{0}=\left(0,0,0,0, \omega^{A_{0}^{(0)}}\right)$, where $\omega^{A_{0}^{(0)}}$ is an arbitrary function. Thus, by using this mode, we obtain the following constraint

$$
\Omega^{0}=v_{i}^{0} \frac{\delta V^{0}}{\delta \xi^{i}} \rightarrow \partial_{i} \Pi_{(0)}^{i}+P_{(0)}=0,
$$

we can observe that this constraint is the secondary constraint obtained by means Dirac's method given in (13). We would comment that in [FJ] framework, there are not Dirac's primary constraints as expected. Let us calculate if there are present more constraints in the context of [FJ]. In order to archive this aim, we write in matrix form the following system [23]

$$
\begin{aligned}
f_{i j}^{0} \dot{\xi}^{j} & =\frac{\delta V^{0}}{\delta \xi}, \\
\frac{\delta \Omega^{0}}{\delta \xi^{i}} \dot{\xi}^{i} & =0,
\end{aligned}
$$

by using the symplectic variables and $V^{0}$, that matrix has the explicit form

$$
F_{i j}(x, y)=\left(\begin{array}{ccccc}
0 & -\delta_{j}{ }^{i} & 0 & 0 & 0 \\
\delta_{j}{ }^{i} & 0 & 0 & 0 & 0 \\
0 & 0 & 0 & -1 & 0 \\
0 & 0 & 1 & 0 & 0 \\
0 & 0 & 0 & 0 & 0 \\
0 & \partial_{i} & 0 & 1 & 0
\end{array}\right) \delta^{3}(x-y)
$$

thus, we can observe that (34) is not a squared matrix as is expected, however, it has a mode given by $\left(v^{1}\right)_{i}^{T}=\left(-\partial_{i} \omega^{A_{i}^{(0)}}, 0, \omega^{A_{i}^{(0)}}, 0,0, \omega^{A_{i}^{(0)}}\right)$. This mode, is used in order to obtain more constraints, thus, we calculate the following contraction [23]

$$
\left(v^{1}\right)_{i}^{T} Z_{i}=0
$$

where

$$
Z_{i}=\left(\begin{array}{c}
\frac{\delta V^{0}}{\delta \xi^{i}} \\
0
\end{array}\right) .
$$

By performing the contraction with the mode $\left(v^{1}\right)_{i}^{T}$, we find that (35) is an identity, therefore, in $[\mathrm{FJ}]$ framework there are not more constraints for the theory under study.

By following with the method, in order to construct a new symplectic Lagrangian containing the information of the constraint obtained in (32), we introduce a Lagrangian multiplier associated to the constraint $\Omega^{0}$, namely $\rho^{(0)}$, and we obtain the following symplectic Lagrangian

$$
\mathcal{L}^{1}=\Pi_{(0)}^{i} \dot{A}_{i}^{(0)}+P_{(0)} \dot{\theta}^{(0)}-\left(\partial_{i} \Pi_{(0)}^{i}+P_{(0)}\right) \dot{\rho}^{(0)}-V^{1},
$$

where $V^{1}=\left.V^{0}\right|_{\Omega^{0}=0}=\left(\frac{1}{2} \Pi_{(0)}^{i} \Pi_{(0)}^{i}+\frac{1}{4 m^{2}} P_{(0)} P_{(0)}+\frac{1}{4} F_{i j}^{(0)} F_{(0)}^{i j}-m^{2}\left(A_{i}^{(0)}+\partial_{i} \theta^{(0)}\right)^{2}\right)$. Now we will consider $\rho^{(0)}$ as a symplectic variable, thus our new set of symplectic variables 
are given by $\xi^{1 i}=\left(A_{i}^{(0)}, \Pi_{(0)}^{i}, \theta^{(0)}, P_{(0)}, \rho^{(0)}\right)$ and the new symplectic 1 -forms are $a_{i}^{1}=$ $\left(\Pi_{(0)}^{i}, 0, P_{(0)}, 0,-\left(\partial_{i} \Pi_{(0)}^{i}+P_{(0)}\right)\right)$. In this manner, with these symplectic variables, we obtain the following symplectic matrix given by

$$
f_{i j}^{1}(x, y)=\left(\begin{array}{ccccc}
0 & -\delta_{j}{ }^{i} & 0 & 0 & 0 \\
\delta_{j}{ }^{i} & 0 & 0 & 0 & -\partial_{i} \\
0 & 0 & 0 & -1 & 0 \\
0 & 0 & 1 & 0 & -1 \\
0 & \partial_{i} & 0 & 1 & 0
\end{array}\right) \delta^{3}(x-y)
$$

We are able to observe that $f_{i j}^{1}(x, y)$ is singular, however, we have showed that there are not more constraints; the noninvertibility of (38) means that the theory has a gauge symmetry. Hence, we choose the following ( gauge condition) constraint $\Omega^{2}=\partial^{i} A_{i}^{(0)}=0$, and we introduce a new Lagrange multiplier $\eta_{(0)}$ for constructing the following symplectic Lagrangian

$$
\mathcal{L}^{2}=\Pi_{(0)}^{i} \dot{A}_{i}^{(0)}+P_{(0)} \dot{\theta}^{(0)}-\left(\partial_{i} \Pi_{(0)}^{i}+P_{(0)}\right) \dot{\rho}^{(0)}-\left(\partial^{i} A_{i}^{(0)}\right) \dot{\eta}_{(0)}-V^{2},
$$

where $V^{2}=\left.V^{1}\right|_{\Omega^{2}=0}$. From the symplectic Lagrangian (39), we take the following symplectic variables $\xi^{2 i}=\left(A_{i}^{(0)}, \Pi_{(0)}^{i}, \theta^{(0)}, P_{(0)}, \rho^{(0)}, \eta_{(0)}\right)$ and the corresponding symplectic 1-forms are $a_{i}^{2}=$ $\left(\Pi_{(0)}^{i}, 0, P_{(0)}, 0,-\left(\partial_{i} \Pi_{(0)}^{i}+P_{(0)}\right),-\partial^{i} A_{i}^{(0)}\right)$. In this way, by using these symplectic variables, we obtain the following symplectic matrix

$$
f_{i j}^{2}(x, y)=\left(\begin{array}{cccccc}
0 & -\delta_{j}{ }^{i} & 0 & 0 & 0 & -\partial_{i} \\
\delta_{j}{ }^{i} & 0 & 0 & 0 & -\partial_{i} & 0 \\
0 & 0 & 0 & -1 & 0 & 0 \\
0 & 0 & 1 & 0 & -1 & 0 \\
0 & \partial_{i} & 0 & 1 & 0 & 0 \\
\partial_{i} & 0 & 0 & 0 & 0 & 0
\end{array}\right) \delta^{3}(x-y)
$$

we observe that $f_{i j}^{2}(x, y)$ is not singular, hence, it is an invertible matrix. After a long but straightforward calculation, the inverse of $f_{i j}^{2}(x, y)$ is given by

$$
\left(f_{i j}{ }^{2}(x, y)\right)^{-1}=\left(\begin{array}{cccccc}
0 & \delta_{j}{ }^{i}-\frac{\partial^{i} \partial_{j}}{\nabla^{2}} & 0 & 0 & 0 & \frac{\partial^{i}}{\nabla^{2}} \\
-\delta_{j}{ }^{i}+\frac{\partial^{i} \partial_{j}}{\nabla^{2}} & 0 & \frac{\partial^{i}}{\nabla^{2}} & 0 & \frac{\partial^{i}}{\nabla^{2}} & 0 \\
0 & -\frac{\partial^{i}}{\nabla^{2}} & 0 & 1 & 0 & \frac{1}{\nabla^{2}} \\
0 & 0 & -1 & 0 & 0 & 0 \\
0 & -\frac{\partial^{i}}{\nabla^{2}} & 0 & 0 & 0 & \frac{1}{\nabla^{2}} \\
-\frac{\partial^{i}}{\nabla^{2}} & 0 & -\frac{1}{\nabla^{2}} & 0 & -\frac{1}{\nabla^{2}} & 0
\end{array}\right) \delta^{3}(x-y)
$$

Therefore, from (41) it is possible to identify the following [FJ] generalized brackets given by

$$
\left\{\xi_{i}^{2}(x), \xi_{j}^{2}(y)\right\}_{F J}=\left(f_{i j}^{2}(x, y)\right)^{-1} .
$$


Thus, from (41) we obtain

$$
\begin{aligned}
& \left\{A_{i}^{(0)}(x), \Pi_{(0)}^{j}(y)\right\}_{F J}=\left(\delta^{j}{ }_{i}-\frac{\partial^{j} \partial_{i}}{\nabla^{2}}\right) \delta^{3}(x-y), \\
& \left\{P(x)_{(0)}, \theta(y)^{(0)}\right\}_{F J}=-\delta^{3}(x-y), \\
& \left\{\Pi_{(0)}^{i}(x), \theta(y)^{(0)}\right\}_{F J}=\frac{\partial^{i}}{\nabla^{2}} \delta^{3}(x-y),
\end{aligned}
$$

we observe that the generalized [FJ] brackets are equivalent with those given in (26) obtained by using a pure Dirac's method. In addition, the quantization of the theory is done by the replacement of classical [FJ] brackets to commutators

$$
\left\{\xi_{i}^{2}(x), \xi_{j}^{2}(y)\right\}_{F J} \longrightarrow-\frac{i}{\hbar}\left[\widehat{\xi}_{i}^{2}(x), \widehat{\xi}_{j}^{2}(y)\right],
$$

where $\widehat{\xi}_{i}^{2}(x)$ are the quantum operators of the dynamical variables. Therefore, in [FJ] framework, there are the following constraints

$$
\begin{aligned}
& \psi_{1}^{(0)}=\partial^{i} A_{i}^{(0)}=0, \\
& \psi_{2}^{(0)}=\partial_{i} \Pi_{(0)}^{i}+P_{(0)}=0,
\end{aligned}
$$

there are less constraints with respect Dirac's framework as expected; there are not present Dirac's primary constraints. We would remark that all these results are absent in the literature.

\section{FADDIEEV-JACKIW QUANTIZATION FOR THE KK-MODES}

Now we will obtain the generalized [FJ] brackets for the KK-modes. For our aims, from (10) we identify the symplectic Lagrangian for the KK-modes given in the following expression

$$
\mathcal{L}^{0}=\Pi_{(n)}^{i} \dot{A}_{i}^{(n)}+P_{(n)} \dot{\theta}^{(n)}+\Pi_{(n)}^{5} \dot{A}_{5}^{(n)}-V^{0},
$$

where

$$
\begin{aligned}
V^{0}= & \sum_{n=1}^{\infty}\left[\frac{1}{2} \Pi_{(n)}^{i} \Pi_{(n)}^{i}+\frac{1}{4 m^{2}} P_{(n)} P_{(n)}+\frac{1}{4} F_{i j}^{(n)} F_{(n)}^{i j}-A_{0}^{(n)}\left(\partial_{i} \Pi_{(n)}^{i}+\frac{n}{R} \Pi_{(n)}^{5}+P_{(n)}\right)-m^{2}\left(A_{i}^{(n)}+\partial_{i} \theta^{(n)}\right)^{2}\right. \\
& \left.\left.+\frac{1}{2} \Pi_{5}^{(n)} \Pi_{5}^{(n)}+\frac{1}{2}\left(\partial_{i} A_{5}^{(n)}+\frac{n}{R} A_{i}^{(n)}\right)^{2}-m^{2}\left(A_{5}^{(n)}-\frac{n}{R} \theta^{(n)}\right)^{2}\right]\right) .
\end{aligned}
$$

Thus, we identify the following symplectic dynamical variables $\xi^{i}=$ $\left(A_{i}^{(n)}, \Pi_{(n)}^{i}, A_{5}^{(n)}, \Pi_{(n)}^{5}, \theta^{(n)}, P_{(n)}, A_{0}^{(n)}\right)$ and the components of the symplectic 1-forms are $a_{i}=\left(\Pi_{(n)}^{i}, 0, \Pi_{(n)}^{5}, 0, P_{(n)}, 0,0\right)$. In this manner, we obtain the following symplectic matrix

$$
f_{i j}^{0}(x, y)=\left(\begin{array}{ccccccc}
0 & -\delta_{j}{ }^{i} & 0 & 0 & 0 & 0 & 0 \\
\delta_{j}{ }^{i} & 0 & 0 & 0 & 0 & 0 & 0 \\
0 & 0 & 0 & -1 & 0 & 0 & 0 \\
0 & 0 & 1 & 0 & 0 & 0 & 0 \\
0 & 0 & 0 & 0 & 0 & -1 & 0 \\
0 & 0 & 0 & 0 & 1 & 0 & 0 \\
0 & 0 & 0 & 0 & 0 & 0 & 0
\end{array}\right) \delta^{3}(x-y),
$$


we observe that this matrix is singular, and has the following mode $v^{0}=\left(0,0,0,0,0,0, \omega^{A_{0}^{(n)}}\right)$, where $\omega^{A_{0}^{(n)}}$ is an arbitrary function. Thus, by using this vector, we obtain the following constraint

$$
\Omega_{(n)}^{0}=v_{i}^{0} \frac{\delta V^{0}}{\delta \xi^{i}} \rightarrow \partial_{i} \Pi_{(n)}^{i}+\frac{n}{R} \Pi_{(n)}^{5}+P_{(n)}=0,
$$

this constraint corresponds to the secondary constraint obtained by Dirac's method. Now, we will compute if there are more constraints. In fact, by considering (47) and (48) into (33), we find that for the KK-modes the matrix (33) takes the form

$$
F_{i j}(x, y)=\left(\begin{array}{ccccccc}
0 & -\delta_{j}{ }^{i} & 0 & 0 & 0 & 0 & 0 \\
\delta_{j}{ }^{i} & 0 & 0 & 0 & 0 & 0 & 0 \\
0 & 0 & 0 & -1 & 0 & 0 & 0 \\
0 & 0 & 1 & 0 & 0 & 0 & 0 \\
0 & 0 & 0 & 0 & 0 & -1 & 0 \\
0 & 0 & 0 & 0 & 1 & 0 & 0 \\
0 & 0 & 0 & 0 & 0 & 0 & 0 \\
0 & \partial_{i} & 0 & \frac{n}{R} & 0 & 1 & 0
\end{array}\right) \delta^{3}(x-y),
$$

that matrix has a mode given by $\left(v^{1}\right)_{i}^{T}=\left(-\partial_{i} \omega^{A_{i}^{(n)}}, 0,-\frac{n}{R} \omega^{A_{i}^{(n)}}, 0,-\omega^{A_{i}^{(n)}}, 0,0,-\omega^{A_{i}^{(n)}}\right)$, by using this mode in (35), we obtain an identity. Therefore, there are not more constraints for the KKmodes.

With all that information obtained at the moment, we introduce $\rho^{(n)}$ as a Lagrangian multiplier associated for the constraint $\Omega_{(n)}^{0}$, thus, we construct a new Lagrangian given by

$$
\mathcal{L}^{1}=\Pi_{(n)}^{i} \dot{A}_{i}^{(n)}+P_{(0)} \dot{\theta}^{(n)}+\Pi_{(n)}^{5} \dot{A}_{5}^{(n)}-\left(\partial_{i} \Pi_{(n)}^{i}+\frac{n}{R} \Pi_{(n)}^{5}+P_{(n)}\right) \dot{\rho}^{(n)}-V^{1},
$$

where

$$
\begin{aligned}
V^{1}=\left.V^{0}\right|_{\Omega^{0}=0} & =\sum_{n=1}^{\infty}\left[\frac{1}{2} \Pi_{(n)}^{i} \Pi_{(n)}^{i}+\frac{1}{4 m^{2}} P_{(n)} P_{(n)}+\frac{1}{4} F_{i j}^{(n)} F_{(n)}^{i j}\right. \\
& -m^{2}\left(A_{i}^{(n)}+\partial_{i} \theta^{(n)}\right)^{2}+\frac{1}{2} \Pi_{5}^{(n)} \Pi_{5}^{(n)}+\frac{1}{2}\left(\partial_{i} A_{5}^{(n)}+\frac{n}{R} A_{i}^{(n)}\right)^{2} \\
& \left.-m^{2}\left(A_{5}^{(n)}-\frac{n}{R} \theta^{(n)}\right)^{2}\right] .
\end{aligned}
$$

Now we will consider to $\rho^{(n)}$ as a symplectic variable and our new set of variables are given by $\xi^{i}=\left(A_{i}^{(n)}, \Pi_{(n)}^{i}, A_{5}^{(n)}, \Pi_{(n)}^{5}, \theta^{(n)}, P_{(n)}, \rho^{(n)}\right)$ and the new symplectic 1-forms are $a_{i}=$ $\left(\Pi_{(n)}^{i}, 0, \Pi_{(n)}^{5}, 0, P_{(n)}, 0,-\left(\partial_{i} \Pi_{(n)}^{i}+\frac{n}{R} \Pi_{(n)}^{5}+P_{(n)}\right)\right)$. In this manner, by using these symplectic vari- 
ables, we obtain the following symplectic matrix

$$
f_{i j}^{1}(x, y)=\left(\begin{array}{ccccccc}
0 & -\delta_{j}{ }^{i} & 0 & 0 & 0 & 0 & 0 \\
\delta_{j}{ }^{i} & 0 & 0 & 0 & 0 & 0 & -\partial_{i} \\
0 & 0 & 0 & -1 & 0 & 0 & 0 \\
0 & 0 & 1 & 0 & 0 & 0 & -\frac{n}{R} \\
0 & 0 & 0 & 0 & 0 & -1 & 0 \\
0 & 0 & 0 & 0 & 1 & 0 & 0 \\
0 & \partial_{i} & 0 & \frac{n}{R} & 0 & 0 & 0
\end{array}\right) \delta^{3}(x-y)
$$

we are able to observe that $f_{i j}^{1}(x, y)$ is not an invertible matrix, this means that the theory has a gauge symmetry. Hence, in order to obtain the results given in (28) we chose the following constraint condition $\Omega^{2(n)}=A_{5}^{(n)}=0$. The lector could ask; why it is not taken into account the gauge $\Pi_{5}^{(n)}+\frac{n}{R} A_{0}^{(n)}$ as is used in Dirac's method?. The answer is that with that gauge, the matrix $f_{i j}^{1}(x, y)$ is not invertible, because we do not expect more constraints, then the [FJ] formalism must ends; the constraint $\Omega^{2(n)}$ makes invertible to $f_{i j}^{1}(x, y)$ and this allows us to finish with our analysis. Hence, by introducing a new Lagrange multiplier associated to $\Omega^{2(n)}$ we construct the following symplectic Lagrangian

$$
\mathcal{L}^{2}=\Pi_{(n)}^{i} \dot{A}_{i}^{(n)}+P_{(0)} \dot{\theta}^{(n)}+\Pi_{(n)}^{5} \dot{A}_{5}^{(n)}-\left(\partial_{i} \Pi_{(n)}^{i}+\frac{n}{R} \Pi_{(n)}^{5}+P_{(n)}\right) \dot{\rho}^{(n)}-A_{5}^{(n)} \dot{\eta}_{(n)}-V^{2},
$$

where

$$
\begin{aligned}
V^{2}=\left.V^{1}\right|_{\Omega^{2(n)}=0} & =\sum_{n=1}^{\infty}\left[\frac{1}{2} \Pi_{(n)}^{i} \Pi_{(n)}^{i}+\frac{1}{4 m^{2}} P_{(n)} P_{(n)}+\frac{1}{4} F_{i j}^{(n)} F_{(n)}^{i j}\right. \\
& \left.-m^{2}\left(A_{i}^{(n)}+\partial_{i} \theta^{(n)}\right)^{2}+\frac{1}{2}\left(\frac{n}{R} A_{i}^{(n)}\right)^{2}-m^{2}\left(\frac{n}{R} \theta^{(n)}\right)^{2}\right]
\end{aligned}
$$

Therefore, we identify the following symplectic variables $\xi^{i}=$ $\left(A_{i}^{(n)}, \Pi_{(n)}^{i}, A_{5}^{(n)}, \Pi_{(n)}^{5}, \theta^{(n)}, P_{(n)}, \rho^{(n)}, \eta_{(n)}\right) \quad$ and $\quad$ the $\quad$ corresponding 1 -forms $a_{i}=$ $\left(\Pi_{(n)}^{i}, 0, \Pi_{(n)}^{5}, 0, P_{(n)}, 0,-\left(\partial_{i} \Pi_{(n)}^{i}+\frac{n}{R} \Pi_{(n)}^{5}+P_{(n)}\right),-A_{5}^{(n)}\right) . \quad$ Thus, by using these symplectic variables we obtain the following symplectic matrix

$$
f_{i j}^{2}(x, y)=\left(\begin{array}{cccccccc}
0 & -\delta_{j}{ }^{i} & 0 & 0 & 0 & 0 & 0 & 0 \\
\delta_{j}{ }^{i} & 0 & 0 & 0 & 0 & 0 & -\partial_{i} & 0 \\
0 & 0 & 0 & -1 & 0 & 0 & 0 & -1 \\
0 & 0 & 1 & 0 & 0 & 0 & -\frac{n}{R} & 0 \\
0 & 0 & 0 & 0 & 0 & -1 & 0 & 0 \\
0 & 0 & 0 & 0 & 1 & 0 & -1 & 0 \\
0 & \partial_{i} & 0 & \frac{n}{R} & 0 & 1 & 0 & 0 \\
0 & 0 & 1 & 0 & 0 & 0 & 0 & 0
\end{array}\right) \delta^{3}(x-y)
$$


We observe that this matrix is not singular, therefore we can calculate its inverse. The inverse of the matrix $f_{i j}^{2}(x, y)$ is given by

$$
\left(f_{i j}^{2}(x, y)\right)^{-1}=\left(\begin{array}{cccccccc}
0 & \delta_{j}{ }^{i} & 0 & -\frac{R}{n} \partial^{i} & 0 & 0 & 0 & \frac{R}{n} \partial^{i} \\
-\delta_{j}{ }^{i} & 0 & 0 & 0 & 0 & 0 & 0 & 0 \\
0 & 0 & 0 & 0 & 0 & 0 & 0 & 1 \\
\frac{R}{n} \partial^{i} & 0 & 0 & 0 & \frac{R}{n} & 0 & \frac{R}{n} & 0 \\
0 & 0 & 0 & -\frac{R}{n} & 0 & 1 & 0 & \frac{R}{n} \\
0 & 0 & 0 & 0 & -1 & 0 & 0 & 0 \\
0 & 0 & 0 & -\frac{R}{n} & 0 & 0 & 0 & \frac{R}{n} \\
-\frac{R}{n} \partial^{i} & 0 & -1 & 0 & -\frac{R}{n} & 0 & -\frac{R}{n} & 0
\end{array}\right) \delta^{3}(x-y),
$$

In this manner, from (53) we obtain the following generalized [FJ] brackets among the physical fields

$$
\begin{aligned}
\left\{A_{i}^{(n)}(x), \Pi_{(n)}^{j}(y)\right\}_{F J} & =\delta^{j}{ }_{i} \delta^{3}(x-y), \\
\left\{\Pi_{(n)}^{5}(x), A_{i}^{(n)}(y)\right\}_{F J} & =\frac{R}{n} \partial_{i} \delta^{3}(x-y), \\
\left\{\theta^{(n)}(x), P_{(n)}(y)\right\}_{F J} & =\delta^{3}(x-y),
\end{aligned}
$$

where we can observe that these brackets are the same with those obtained in (28) by means of Dirac's framework. It is important to comment that in Dirac's procedure, $A_{5}^{(n)}$ are identified as pseudoGoldston bosons; in [FJ] formalism the fields $A_{5}^{(n)}$ are identified as constraints for the theory, this gauge allowed us end our $[\mathrm{FJ}]$ analysis. Hence, in [FJ] method we obtain for the KK-modes the following constraints

$$
\begin{aligned}
\partial_{i} \Pi_{(n)}^{i}+\frac{n}{R} \Pi_{(n)}^{5}+P_{(n)} & =0 \\
A_{5}^{(n)} & =0 .
\end{aligned}
$$

Therefore, we have obtained the quantum brackets for the zero and for KK-modes by means two different approaches.

\section{CONCLUSSIONS AND PROSPECTS}

In this paper, the Hamiltonian and the [FJ] analysis for a five-dimensional Stüeckelberg's theory in the context of extra dimensions has been performed. Respect to the Hamiltonian formalism, we obtained the complete canonical description of the theory. After performing the compactification of the fifth dimension on a $S^{1} / \mathbf{Z}_{\mathbf{2}}$ orbifold, we found that the theory is composed by a four-dimensional Stüeckelberg theory identified with the zero-mode plus a tower of KK-modes. We report the complete constraints program, we found that the theory has only first class constraints. From the gauge transformations of the theory and fixing a particular gauge for the gauge parameters, we identified massive vector fields, massive scalar fields and the fields $A_{5}^{(n)}$ are identified as pseudo-Goldston 
bosons just as is present in five-dimensional Maxwell theory [20]. Furthermore, we constructed the fundamental Dirac's brackets of the zero modes and the Dirac's brackets for the KK-modes.

On the other hand, we performed the $[\mathrm{FJ}]$ quantization for the theory under study. We calculate the generalized [FJ] brackets and we showed that both [FJ] brackets and Dirac's brackets are the same. We found in the context of [FJ] that the fields $A_{5}^{(n)}$ are identified as constraints of the theory, and they are not absorbed as it is present in Dirac's method being identified as pseudo-Goldston bosons. Furthermore, we could observe that we arrived to the constraints and the generalized brackets in less steps than Dirac's method, this means that [FJ] is in particular, for the theory under study, more economic than Dirac's procedure 24]. In this manner, we have stablished all the elements for studying the quantization aspects, for example, we can use Dirac's brackets or $[\mathrm{FJ}]$ generalized brackets for studying the observables of the theory that could be amenable to test. On the other hand, by using those brackets we also could calculate the propagators among gauge fields and carryout the quantization by means of canonical approach or by means [FJ] framework, however, all these ideas are in progress and will be the subject of forthcoming works.

\section{Acknowledgements}

This work was supported by CONACyT under Grant No. CB-2010/157641. We would like to thank R. Cartas-Fuentevilla for discussion on the subject and reading the manuscript.

[1] A. Proca, J. de Phys. et le Radium 7, 347-353, (1936)

[2] N. Banerjee, R. Banerjee, Mod.Phys.Lett. A11, 1919-1928, (1996).

[3] Soon-Tae Hong, Yong-Wan Kim, Young-Jai Park and K.D. Rothe, Mod.Phys.Lett. A17, 435-452, (2002).

[4] E. Stueckelberg, Helv. Phys. Acta 11, 299-312. (1938).

[5] Boris Kors, Pran Nath, JHEP 0507:069, (2005)

[6] W. Pauli, Rev. Mod. Phys. 13, 203-232. (1941).

[7] H. Ruegg, M. Ruiz-Altaba, Int. J. Mod.Phys. A 19:3265-3348, (2004)

[8] A. H. Chamseddine, Phys. Rev. D 243065 (1981); E. Witten, Phys. Lett. B 155, 15, (1985); C. P. Burgess, A. Font and F. Quevedo, Nucl. Phys. B 272, 661, (1986); S. Ferrara, C. Kounnas and M. Porrati, Phys. Lett. B 181, 263, (1986) .

[9] C. Marshall, and P. Ramond, Nucl. Phys. B85, 375-414, (1975).

[10] M. Kalb and P. Ramond, Phys. Rev. D9, 2273-2284, (1974).

[11] T. J. Allen, M. J. Bowick and A. Lahiri, Mod. Phys. Lett. A 6, 559, (1991).

[12] M. Gogberashvili, A. Herrera-Aguilar, D. Malagón-Morejón and R. R. Mora-Luna, Phys.Lett. B 725, 208-211, (2013).

[13] G. Weiglein et al. (Physics Interplay of the LHC and the ILC), arXiv:hep-ph/0410364

[14] M. Henneaux and C. Teitelboim, Quantisation of gauge systems, Princeton University Press, Princeton,(1992).

[15] A. Escalante and L. Carbajal, Ann. Phys. 326, 323179, (2011).

[16] A. Escalante and I. Ruvalcaba-García, Int. J. Geom. Methods Mod. Phys. 9, 1250053(2012). 
[17] A. Escalante and J. Manuel-Cabrera, Ann. Phys. 343, 2739, (2014).

[18] L. Faddeev and R. Jackiw, Phys. Rev. Lett. 60, 1692 (1988).

[19] A. Muck, A. Pilaftsis and R. Ruckl, Phys. Rev. D 65, 085037 (2002).

[20] A. Perez-Lorenzana, J. Phys. Conf. Ser. 18, 224 (2005).

[21] H. Novales-Sanchez and J. J. Toscano, Phys. Rev. D, 82, 116012 (2010).

[22] E. Harikumar and M. Sivakumar, Mod.Phys.Lett. A15, 121-132, (2000).

[23] L. Liao and Y. C. Huang, Ann. Phys. 322, 24692484, (2007).

[24] J. A. Garcia and J.M.Pons, Int. J. Mod. Phys. A 12, 451, (1997). 